\title{
Entre interview littéraire et entretien d'écrivain : Orhan Pamuk dans la presse française
}

Between the Literary and the Author's Interview: Orhan Pamuk in the French Press

\section{Adeline Wrona}

\section{(2) OpenEdition}

\section{Journals}

Édition électronique

URL : http://journals.openedition.org/aad/1655

DOI : 10.4000/aad. 1655

ISSN : 1565-8961

Éditeur

Université de Tel-Aviv

Référence électronique

Adeline Wrona, «Entre interview littéraire et entretien d'écrivain : Orhan Pamuk dans la presse

française », Argumentation et Analyse du Discours [En ligne], 12 | 2014, mis en ligne le 20 avril 2014 consulté le 23 septembre 2019. URL : http://journals.openedition.org/aad/1655 ; DOI : 10.4000/aad. 1655

Ce document a été généré automatiquement le 23 septembre 2019

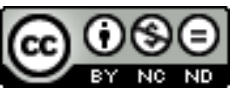

Argumentation \& analyse du discours est mis à disposition selon les termes de la licence Creative Commons Attribution - Pas d'Utilisation Commerciale - Pas de Modification 4.0 International. 


\section{Entre interview littéraire et entretien d'écrivain : Orhan Pamuk dans la presse française}

Between the Literary and the Author's Interview: Orhan Pamuk in the French

Press

Adeline Wrona

1 Orhan Pamuk, écrivain turc lauréat du prix Nobel de Littérature en 2006, vit depuis 2005 entre Istanbul et New York; ses ouvrages sont traduits dans plus de quarante langues. En France, Pamuk est un « auteur Gallimard », maison prestigieuse qui a publié depuis 1988 l'intégralité de ses douze livres traduits ; il fut aussi l'invité, à l'automne 2012, du Musée du Louvre, qui lui donna «Carte blanche» pour accompagner l'ouverture des nouvelles salles du département des Arts de l'Islam.

2 Si nous avons choisi d'analyser le genre de l'entretien littéraire à travers l'exemple d'Orhan Pamuk, c'est parce que la notoriété acquise par cet auteur s'avère à la fois incontestable et complexe. La reconnaissance internationale consacre en effet l'œuvre d'un auteur devenu indésirable dans son propre pays, où il est l'objet de menaces politiques ; or c'est un entretien publié en 2005 dans un journal suisse, et la multiplicité de ses reprises médiatiques, qui a suscité l'une et, au moins en partie, l'autre de ces conséquences.

3 Le « cas Pamuk » constitue de ce fait un exemple intéressant pour qui s'interroge sur le rôle joué par les interviews médiatiques dans l'édification et la mise en circulation des « images d'auteur ", selon l'expression proposée par de récents travaux en analyse du discours, et en particulier par Ruth Amossy (Amossy 2009) et Dominique Maingueneau (Maingueneau 2004 et 2009). Dans le cas des entretiens, l'image d'auteur paraît en l'occurrence devoir être questionnée à deux niveaux : il s'agit à la fois de la figure de l'écrivain et de celle du journaliste. Les modes de textualisation et d'éditorialisation de l'entretien littéraire mettent en effet en jeu la rivalité opposant, dans tout entretien journalistique, l'interviewer et l'interviewé ; c'est le «droit régalien du journaliste sur 
l'interviewé ", évoqué par Barthes dans le cours sur Le Neutre (Barthes $2002: 146)$. Ce rapport de force se trouve, dans le cas des entretiens littéraires, compliqué par un enjeu supplémentaire: il s'agit ici d'un face à face entre deux professionnels de l'écriture. Si l'écrivain réactive un travail de présentation de soi à chaque nouvelle interview, on peut penser que le journaliste, de son côté, éprouve dans cet exercice la tension toujours latente entre la professionnalisation d'une pratique d'écriture très normée, d'une part, et l'aspiration à une posture littéraire, d'autre part, qui rompt avec les codes du discours d'information pour revenir à un journalisme de plume.

\section{Un genre ou des genres : qu'est-ce qu'un entretien littéraire?}

Nous avons réuni pour cette analyse un corpus de 19 articles parus dans la presse française, entre janvier 1999 et août 2013 ; tous transcrivent des propos tenus par Orhan Pamuk lors d'un échange avec un journaliste délégué par la rédaction ${ }^{1}$.

Ces textes répondent donc, en première analyse, à la définition de l'entretien proposée par Yanoshevsky (se référant à Benveniste et à Kerbrat-Orecchioni) : "[L'entretien] consiste en un dialogue qui se déroule en face-à-face, et où se produit entre les interlocuteurs un véritable échange qui détermine de façon réciproque et continue les comportements des partenaires en présence » $(2006: 150)$. Ils sont aussi conformes à la description plus sibylline de Philippe Lejeune: "Réponse à un questionnement, intention de parler à un public donné représenté par le questionneur, publication quasi-immédiate » (Lejeune 1980, cité par Seillan 2004). Pourtant, au-delà de ce trait commun, les caractéristiques de ces articles font apparaître de grandes diversités, qui tiennent à la fois aux dispositifs énonciatifs, à l'organisation typographique, au rubriquage, enfin à tout ce qui définit l'éditorialisation et la matérialité du discours journalistique. Comment alors rendre compte de cette hétérogénéité ? Et peut-on s'appuyer sur ces choix éditoriaux pour interpréter la place occupée par l'écrivain dans l'espace médiatique?

$6 \quad$ Faire de l'entretien littéraire un genre homogène ne rendrait pas compte de la variété de ses appropriations possibles; Galia Yanoshevsky propose plutôt de penser l'entretien comme "une forme générique riche et productive " (Yanoshevsky 2006: 148), qui recourt à différents dispositifs énonciatifs. De fait, cette diversité des pratiques s'observe aussi dans l'évolution du genre qui, depuis son apparition dans la presse française à la fin $\mathrm{du} 19^{\mathrm{e}}$ siècle, est le lieu d'une mise en tension entre modèles d'écriture littéraires et aspiration à une professionnalisation des discours journalistiques.

$7 \quad$ Les premiers entretiens se déploient dans le sillage du reportage ; il s'agit, rapporte par exemple Pierre Giffard en 1880, de "dépêcher un journaliste auprès d'un personnage quelconque, momentanément mis en vue par les circonstances, qu'il soit homme politique, homme de lettres, savant ou homme d'épée, et cela sans le connaître le moins du monde ", le tout "dans le but d'obtenir de la bouche même de cette illustration le plus de renseignements possibles sur son compte » (Lavaud \& Thérenty 2006 : 9). Genre de l'immédiateté, reposant sur une promotion de la rencontre en chair et en os des acteurs de l'actualité, l'interview est perçue, à ses débuts, comme «le mode le plus radical de l'information pour l'information» (Seillan 2002:16)². On ne s'éloigne pas 
vraiment, toutefois, de l'univers de la littérature; dès les premières enquêtes systématiques menées par Jules Huret pour Le Figaro, la rencontre avec l'écrivain fait partie des attendus du genre, dotant ce format médiatique naissant d'une légitimité dérivée de la personnalité rencontrée : « homme de lettres, savant ou homme d'épée », écrivait plus haut Giffard, sont les " personnages quelconques» que les « circonstances» mettent au premier plan de l'actualité. Selon Dorothy Speirs, Émile Zola est pendant deux décennies « l'homme le plus interviewé de France » (Speirs 1990 : 302).

8 La qualité «littéraire » de l'entretien doit-elle alors être conçue comme liée à la personne rencontrée, ou bien peut-on y entendre une qualification de l'écriture journalistique? Jules Huret, au tournant du $20^{\mathrm{e}}$ siècle, ou Frédéric Lefèvre dans l'entredeux guerres (pour Les Nouvelles littéraires), œuvrent à la défense et illustration d'une écriture littéraire de la conversation, qui se conçoit avec le souvenir et les méthodes du dialogue romanesque. Si l'on veut éviter que l'interviewer ne soit «un vulgaire perroquet ", estime Zola, alors il faudrait « confier les interviews à des têtes de ligne, à des écrivains de premier ordre, des romanciers extrêmement habiles, qui, eux, sauraient tout remettre au point » (Zola 1893).

Le genre de l'entretien s'avère bien tendu entre différents modèles d'écriture. De fait, à mesure que le journalisme devient une profession autonome, pourvue à la fois d'instances de représentation, de lieux de formation, et de figures consacrées, l'interview se dote de normes rédactionnelles spécifiques, fixées dans des manuels; l'alternance des voix, les modes d'énonciation y sont codifiés selon des protocoles fort éloignés des procédés de fictionnalisation et d'enchâssement narratif qui caractérisaient les pratiques des premiers interviewers.

\section{Entretien littéraire versus interview d'écrivain}

10 La nature « littéraire » de l'entretien pourrait alors être décrite selon deux modalités dominantes, dont nous empruntons la formulation à Jean-Marie Seillan. Éditeur des interviews de Huysmans, et confronté de ce fait à des questions génériques et à des « difficultés d'ordre définitionnel », Seillan (2004) propose de différencier deux cas de figure : dans un premier cas, on parlera «d'entretien littéraire ", pour désigner les articles dont la littérature est proprement l'objet; mais on préférera la formule «d'interview d'écrivain» quand c'est le «sujet parlant» qui confère sa qualité littéraire à une conversation évoquant d'autres sujets que le livre. Car l'écrivain n'échappe pas plus que les autres figures publiques à cette loi qui veut que, dès les années 1890, «tous sont interpellés et sur tout, quelquefois sur ce qu'ils savent, le plus souvent sur ce qu'ils ignorent", selon la formulation proposée par l'écrivain et journaliste Jules Case dans Le Figaro du 2 septembre 1892 (cité par Speirs 1990 : 301).

11 Nous proposons de reprendre à notre compte cette catégorisation, et de l'investir de nouveaux éléments de définition, afin de mettre en visibilité deux formes de logiques médiatiques à l'œuvre dans les articles de notre corpus. Une telle partition constitue un mode opératoire pour dépasser l'extrême hétérogénéité des entretiens réunis, tout en prenant acte de la présence de traits sémiotiques récurrents; et nous faisons le pari que ces récurrences peuvent être référées à une conception particulière des rôles assumés par les deux interlocuteurs, le journaliste et l'écrivain. 
12 Nous différencions un premier groupe d'entretiens, les "entretiens littéraires ", qui, pour reprendre les termes de Jean-Marie Seillan, sont définis par «l'objet dont il est parlé et qui est littérature" (Seillan, 2004). À cette unité thématique (la littérature comme « objet » de discours), nous associons un dispositif d'énonciation caractérisé par la souplesse, voire le flou, des signes de distinction entre les voix de l'interviewer et de l'interviewé. L'alternance entre questions et réponses n'est pas restituée par les formes du dialogue (tirets, paragraphes, discours direct), mais intégrée dans un continuum. Ces articles maximalisent la mixité constitutive du genre de l'interview, décrite par le même Seillan comme "à la fois mimésis et diégésis ", le discours de l'écrivain étant dans ce premier cas « enchâssé » dans un texte continu de tonalité descriptive.

13 Un second groupe, ou une autre variante d'entretiens correspond au modèle de "l'interview d'écrivain ", qui se définit selon Seillan "par le sujet parlant, susceptible d'aborder n'importe quelle question ». La qualité d'écrivain de l'interviewé prime sur le contenu de l'entretien, qui relève du tout venant propre à une actualité non spécifiquement littéraire. À cette détermination par l'identité de l'interviewé, en l'absence de caractérisation des sujets abordés, nous associons des traits formels: les "interviews d'écrivains" regrouperont les interviews de notre corpus qui se caractérisent par la mise en scène explicite d'une alternance des voix, celles de l'interviewer et de l'interviewé, dans un jeu de questions-réponses clairement identifiées.

Nous combinons donc, on le voit, des critères d'ordre thématique (selon que la littérature est ou non l'objet à proprement parler de l'entretien), et des caractéristiques d'ordre sémiotique, relevant de ce qu'Emmanuël Souchier appelle l'énonciation éditoriale (Souchier 1998); l'articulation entre ces deux catégorisations n'est pas tout à fait symétrique, puisque la première famille d'entretiens, conformément à la proposition de Seillan, renvoie prioritairement au contenu de l'entretien, quand la seconde réfère surtout à l'identité de l'interviewé (le contenu pouvant renvoyer à «n'importe quoi »).

Pourtant, cette bipartition heuristique n'est pas si éloignée de celle à laquelle parviennent les auteurs d'un article récemment consacré aux entretiens donnés par Annie Ernaux dans la presse française. Les deux auteurs de ce travail, qui analyse plus spécifiquement « la pratique de l'auto-citation chez Annie Ernaux » (Lopez \& Romeral 2006) distinguent, parmi leur corpus de 26 entretiens, deux grands groupes: l'un réunissant ce qu'ils appellent les "entretiens quantitatifs", l'autre, «les entretiens qualitatifs». Les entretiens quantitatifs répondent à un "objectif prédominant»: "fournir des informations d'actualité». Les entretiens qualitatifs ont pour leur part " principalement en vue la recréation de la personnalité de l'interviewé » (Lopez \& Romeral 2006), et s'il y a apport informatif, le «souci principal » demeure «la qualité esthétique de l'information». L'accent porté sur la "personnalité » de l'écrivain s'associe donc à une visée "esthétique » de l'article journalistique - accentuation qui place l'auteur de l'article en position de visibilité renforcée. De fait, notent les auteurs de cet article, «le lecteur » de cette seconde catégorie de textes « assume dès le début de la lecture l'autorité subjective de l'intervieweur ».

Quelles que soient les dénominations choisies, on conçoit qu'une analyse des interviews données par les écrivains dans les médias rencontre au détour de ses typologies la question de l'équilibre des voix mises en dialogue. Notre démonstration vise à associer cette prise en compte du rapport de force énonciatif entre interviewer et interviewé, à 
une interprétation du rôle respectif du journaliste et de l'écrivain dans l'espace public, et même plus largement de l'articulation entre littérature et politique.

Les modes de circulation et de reprise de la parole auctoriale sont investis dans le cas d'Orhan Pamuk d'un enjeu particulièrement vif : nous l'avons évoqué plus haut, à la suite d'un entretien accordé en février 2005 au supplément hebdomadaire (Das Magazin) d'un quotidien suisse alémanique, Tages-Anzeiger, l'écrivain se voit poursuivi par la justice turque, et menacé de mort dans son pays par des factions ultranationalistes. C'est une phrase isolée de son contexte - une de ces "phrases sans texte", selon la formule proposée par Dominique Maingueneau (Maingueneau 2006) - qui provoque ces réactions. Voici la traduction littérale que l'on peut proposer de cette déclaration, originellement publiée en allemand: «Un million d'Arméniens ont été tués ici [en Turquie]. Et 30000 Kurdes. Mais presque personne n'ose le rappeler $»^{3}$. La circulation médiatique de ces propos, et la persécution qui s'ensuit, conduisent Pamuk à quitter son pays quelques mois plus tard, le dotant d'une stature internationale contrainte et paradoxale. En 2006, l'attribution au romancier du prix Nobel de Littérature peut être considérée comme une conséquence, au moins indirecte, de cette consécration médiatique ambivalente.

Entre entretien littéraire et interview d'écrivain, les entretiens accordés par Orhan Pamuk à la presse française donnent à lire les flottements auxquels se prête un genre journalistique finalement peu stabilisé. L'interview de 2005 ne cesse de faire retour dans les entretiens ultérieurs, au point que chacun des articles publiés après cette date, dans le corpus que nous avons retenu, paraît reprendre à nouveaux frais un travail de réinterprétation du rôle politique de la littérature.

\section{L'entretien littéraire ou la parole enchâssée}

19 Un premier groupe de textes offre toutes les caractéristiques de l'entretien littéraire : Orhan Pamuk y est interrogé sur sa production romanesque, ses sources et inspirations, ses attentes et projets pour l'avenir. La littérature se voit placée au premier plan, et de fait, c'est elle qui « fait » l'actualité, à l'occasion par exemple de la parution française d'un ouvrage qui, déjà en Turquie, aurait connu un succès public. Ces interviews littéraires sont pourtant le lieu de manipulations discursives, qui ont trait à la fois aux modalités de citations de la parole auctoriale, et à la réactivation d'une rivalité séculaire entre l'écrivain et le journaliste.

\subsection{Modalités de citation : paroles migrantes}

L'interview fait partie des genres journalistiques éprouvés, encadrés à la fois par une routine professionnelle et des dispositions juridiques. Les étudiants en école de journalisme assimilent dès leurs premiers mois de formation les recettes de cette rubrique clé dans les médias de presse écrite : des manuels sont à leur disposition, qui conseillent par exemple au journaliste "d'éviter deux extrêmes, la passivité ou la suractivité » dans la conduite de l'entretien, de ménager « des questions plus courtes que les réponses" car "plus il y a d'informations dans une question, moins il y en a dans la réponse » (Rémond 2007 : 39). En somme, toutes sortes d'outils visant à éviter «l'erreur du débutant, qui se met en valeur dès le début » (Montant 1995 : 82). 
21 Les entretiens littéraires avec Orhan Pamuk sont bien loin de respecter les lois du genre; nous en avons retenu 6 exemples, parus entre 1999 et 2006, dans les pages des journaux suivants : Libération, Le Monde, Le Figaro, et Télérama ${ }^{4}$. Si la parole de l'écrivain s'y fait bien entendre, l'ensemble de l'échange se place sous le signe de la " rencontre ", bien plus que de l'interview. De ce fait, les formats adoptés pour la conversation sont sujets à de nombreuses variations.

22 Sur le plan typographique, tout d'abord: placés entre guillemets, les propos de Pamuk sont parfois distingués par les italiques (Libération, Le Figaro, Le Monde, article du 24 avril 2011), parfois maintenus en caractères romains (Télérama, Le Monde, article du 4 avril 2005). Au sein d'un même titre de presse, on le voit, les normes varient - Le Monde abandonne les italiques entre 2005 et 2011.

Sur le plan volumétrique, ensuite, tout semble permis. Alors que les articles du Monde ne ménagent que de courtes citations, d'une phrase à peine, le magazine Télérama adopte une position extrémiste: les propos de l'écrivain occupent l'intégralité de l'article intitulé «Un ardent désir d'écrire », paru le 25 juillet 2007 à l'occasion de la publication en France d'Istanbul. Souvenirs d'une ville; toutefois, il s'agit d'un montage assez curieux qui répartit les paragraphes sous des intertitres non interrogatifs, correspondant manifestement à la réécriture de questions elles-mêmes éclipsées. "C'est de son travail, et de littérature, qu'Orhan Pamuk souhaite aujourd'hui parler » lit-on dans le chapeau d'introduction de cet échange intitulé « Rencontre » : il reste peu de marques de conversation dans cette organisation textuelle, qui escamote les questions et transforme les réponses en textes suivis, entre guillemets, seule trace d'un dialogue présumé. Sous le premier intertitre, «La vie après le Nobel », voici comment s'opère la prise de parole de l'écrivain :

Ma vie a été bouleversée par la gloire soudaine qui va avec l'attribution du Nobel, les interviews, les sollicitations... Alors, aujourd'hui, je me sens comme un enfant qui veut être seul et tranquille dans sa chambre avec ses jouets. Je veux travailler, écrire, rien d'autre.

\section{2. Le récit d'une rencontre?}

On peut alors s'interroger sur la nature de la rencontre elle-même; en presse écrite, l'entretien se doit de porter les marques de cette confrontation corporelle entre les interlocuteurs, qui donne toute sa légitimité à ce travail de "rapportage de la conversation ", selon la formule employée par Maurice Barrès dans une interview sur l'interview parue le 2 décembre 1892 dans Le Journal (Lavaud \& Thérenty 2006 : 18). Là se trouve la condition pour que cette pratique se légitime comme journalistique, empruntant les voies du reportage. La mise en texte doit donc porter les marques de ce moment, à travers divers protocoles rhétoriques: description de l'ameublement, chronique du voyage menant au "grand homme ", évocation des intonations ou des gestes accompagnant les propos. De ce fait, l'interview relève des «genres corporalisants ", selon la typologie proposée par Roselyne Ringoot et Yvon Rochard (Ringoot \& Rochard 2011: 78) : on doit y découvrir le «corps en mouvement du journaliste ", un « avoir été là » qui authentifie l'échange.

Dans le cas des entretiens littéraires analysés, il semble qu'un déplacement des valeurs journalistiques entraîne la valorisation de l'écriture, voire de la réécriture, au détriment de la mise en scène d'une parole vive. De fait, rien dans l'article de Télérama n'atteste la rencontre réelle avec l'écrivain, qui pourrait, sans que le texte en ait été 
modifié, avoir envoyé ses réponses par courrier. Plus encore, plusieurs articles présents dans notre corpus fragilisent la frontière entre entretien et conférence de presse : tel est le cas d'une série de publications entourant l'événement du prix Nobel, en mai 2006 - Le Figaro raconte «la semaine suédoise d'Orhan Pamuk », les 9 et 10 décembre 2006, en citant des propos dont il est difficile de comprendre les conditions de recueil. En voici un exemple :

Pamuk, courtois, élude les questions sur le génocide arménien, se dit attristé par l'état des relations entre la Turquie et l'Europe, mais reste fidèle à ses engagements. Quelles qu'en soient les conséquences. «Non, le prix Nobel ne me protège d'aucune poursuite judiciaire.» Puis, il prend congé pour répondre à d'autres sollicitations, retrouver sa fille, et se préparer au discours devant l'Académie suédoise...

Le Monde propose une interview dont on apprend, après lecture d'une mention discrète, qu'elle précède de plusieurs mois le moment de sa publication. Au moment de l'ouverture par Orhan Pamuk du Musée de l'innocence, à Istanbul, les comptes-rendus journalistiques parus en France portent de même la trace d'un travail de mise en dialogue opéré sur des propos monologiques, très probablement lors d'une visite de presse organisée à cette occasion. Ainsi de l'article publié le 28 avril 2012 par Guillaume Perrier dans Le Monde, sous le titre "Le musée aux mille pages", dont le premier paragraphe se présente comme suit :

C'est une grande maison peinte d'un rouge sombre, dans le quartier bohème de Çukurcuma. Une bâtisse que le prix Nobel de littérature Orhan Pamuk a achetée pour une bouchée de pain en 1999, "dans le but d'y camper l'histoire d'une famille imaginaire dans un quartier d'Istanbul ", dit-il. Il y a conçu un musée, inauguré et ouvert au public le samedi 28 avril.

Plus loin, d'étranges points de suspension interrompent les citations en italiques, comme si les propos oraux de l'écrivain étaient déjà perçus comme un texte à calibrer :

$\mathrm{Au}$ long du parcours, on retrouve les verres de raki ou de thé que consomme volontiers le héros, les bouteilles de limonade " Meltem », une marque inventée par Pamuk, ou encore une multitude de babioles et de factures provenant de l'hôtel Fatih, lui aussi créé de toutes pièces. «Je veux montrer la porosité de la frontière entre l'imaginaire et le réel. Les objets sont entre le rêve et la réalité. [...] Ce n'est pas un musée qui fige les objets du roman. C'est un lieu poétique et artistique. À petite échelle, c'est un peu un musée de la vie quotidienne urbaine et bourgeoise ", poursuit-il.

\section{3. Rivalités auctoriales}

Quelle est alors la valeur d'actualité de ces entretiens, s'ils ne portent pas la marque d'un dialogue tenu ici et maintenant avec le journaliste signataire de l'article? Tout conduit à penser que l'enjeu réside dans la mise en œuvre d'une auctorialité hybride, mêlant les voix du journaliste et de l'écrivain plus qu'elle ne les distingue. Alors que les normes professionnelles répartissant les voix entre interlocuteurs légitiment l'écriture journalistique, visant un idéal de «neutralité » et donc recourant aux stratégies de l'effacement énonciatif, l'entretien littéraire réactive un autre modèle de journalisme, où l'auteur de l'article manifeste une subjectivité assumée, voire empathique.

La relation dialogique ne se joue plus tant alors entre interviewer et interviewé, qu'entre auteur et auteur - l'auteur-journaliste manipulant avec une grande liberté la parole rapportée de l'auteur-écrivain. On ne s'étonnera pas de noter que trois des signatures présentes dans ce corpus sont celles de journalistes ayant eux-mêmes frayé leur voie dans la littérature. Jean-Baptiste Harang s'entretient avec Orhan Pamuk une 
première fois dans Libération. L'article parait le 14 janvier 1999 sous le titre "La démarche turque "; il alterne verbatim aléatoire d'un dialogue parcellaire et longues phrases peu ponctuées, qui rompent ostensiblement avec les normes discursives du journalisme ; en voici un exemple, qui vise à dresser le portrait littéraire de l'écrivain :

Orhan Pamuk est grand, dégingandé, nerveux, il parle vite et fort, il porte des lunettes et voit le monde de sa fenêtre et de ses livres, il ne descend pas dans l'arène des contingences, ou, lorsqu'il s'y rend, c'est sans sa plume, sans son attirail d'écrivain, sinon son immense notoriété qui donne du poids à ses engagements, il y soutient le peuple kurde, il fut le premier intellectuel musulman à prendre la défense de Salman Rushdie et il vient tout juste de se faire remarquer, à sa grande surprise, en refusant le titre « d'artiste d'État ».

Enchaînant sur ce portrait, vient la citation en italiques des propos de l'écrivain :

Chaque année, on distribue ce titre à trois ou quatre artistes, mais cette fois, ils ont dressé une liste de soixante personnes, j'y figure contre le goût de ceux qui m'ont choisi, uniquement à cause de mon audience internationale, pour crédibiliser une liste médiocre, et bien sûr contre mon propre goût.

Près de 17 ans plus tard, dans les pages d'un numéro spécial du Magazine littéraire consacré aux 10 grandes voix de la littérature étrangère (août 2013) le même Jean-Baptiste Harang signe un article consacré au même Orhan Pamuk, intitulé "La vigie myope d'Istanbul »- même auteur, même sujet, et même article, serait-on tenté de conclure à la lecture d'un texte qui ne s'interdit pas le recyclage: «Pamuk est un grand gaillard, aux longues jambes, aux larges lunettes, qui parcourt Istanbul d'une démarche élégante et très rapide pour que son ombre ne marque pas ».

Or Jean-Baptiste Harang est l'auteur d'une dizaine de livres, dont sept romans. Le même tropisme s'observe chez la journaliste du Monde qui signe l'entretien du 4 avril 2005, Catherine Bedarida - un texte qui alterne brèves citations de l'écrivain rencontré, et réécritures libres de la fiction (l'article porte sur le roman Neige) :

$\mathrm{Au}$ dehors, Paris rayonne dans la chaleur de cet automne estival. Au-dedans, l'écrivain turc Orhan Pamuk, de passage pour la présentation de son dernier roman traduit en français, Neige, frissonne. Dans son monde intérieur, l'imagination surpasse la réalité. Après les formules de politesse propres aux voyageurs - Paris, la beauté, la Seine -, il se transporte au cœur de l'hiver anatolien, là où la neige tombe infiniment, suspendant le temps, les espoirs, les attentes.

Concentré sur la blancheur, Orhan Pamuk parle. Comme sur la page, il y inscrit l'histoire de Kars, une ville réelle, située aux confins orientaux de la Turquie, voisine de l'Arménie. fortement littéraire, comme le confirme l'autoportrait publié par la même Catherine Bedarida dans un blog consacré à l'association qu'elle anime, «Le bout de la langue », où l'auteur se décrit comme « poète, journaliste $»^{5}$.

Journaliste et écrivain : telle est aussi l'identité de la reporter d'occasion déléguée par Le Figaro auprès du nobélisé, en décembre 2006 à Stockholm. Clémence Boulouque a cette année-là déjà publié trois romans. Tel est encore le cas d'une autre signature du Monde, Lila Azam Zanganeh, auteur en 2011 d'un ouvrage très remarqué autour de Nabokov (L'Enchanteur : Nabokov et le bonheur, éditions de l'Olivier) dont l'entretien paru avec Orhan Pamuk dans le quotidien daté du 12 mai 2006 ("Orhan Pamuk: être un artiste libre ») présente de fortes similarités avec celui qu'elle signe un an plus tard pour le magazine de l'université de Columbia (Columbia Magazine, été 2007). D'un texte à l'autre circulent notamment des métaphores frappantes, reprises sans autres 
modifications que la transposition d'une langue à l'autre : « Let's say that in my lifetime I never aspired to the political responsabilities ", confesse Orhan Pamuk dans le second texte, « they suddenly hit me, like something falling off a balcony while you're casually strolling down the street». Le Monde proposait une version française antérieure, curieusement proche, de cette aspiration au désengagement :

Disons que, de ma vie, je n'avais jamais cherché à assumer la plupart des responsabilités politiques qui m’ont brusquement pesé sur les épaules! Mais enfin, en raison de jalousies, de ressentiments, de tabous et de pressions diverses, elles me sont tombées dessus. C'est comme quelque chose qui tomberait d'un balcon, alors que vous marchez dans la rue en toute insouciance.

Littéraires, ces entretiens le sont bien, s'affranchissant de certaines contraintes propres au support périodique - régime d'actualité, effacement énonciatif de la personne du journaliste, primat de la "chose vue», respect des normes formelles organisant l'alternance des voix. La parole de l'écrivain s'y voit toutefois soumise à un puissant travail de réappropriation, dont l'exemple le plus flagrant serait donné par l'étude systématique des modes de citation auxquels s'est prêtée la fameuse et fatidique déclaration de février 2005. L'entretien donné par Orhan Pamuk au supplément hebdomadaire du quotidien Tages-Anzeiger se voit réduit à une seule formule qui condense et dramatise le paragraphe de trois phrases par lesquelles l'écrivain, en toute fin d'interview, répondait à la question du journaliste suisse Peer Teuwsen, «Cherchezvous les difficultés ?» - « Oui, tout le monde devrait faire cela. On a ici assassiné 30000 Kurdes. Et un million d'Arméniens. Et presque personne n'ose le rappeler. Alors moi je le fais. Et c'est pour cela qu'on me déteste $»^{6}$.

Après février 2005, il n'est guère d'entretien qui fasse l'économie du renvoi à cette déclaration, systématiquement écourtée, parfois déformée (la mention des Arméniens précédant celle des Kurdes), dans une seule phrase, ou même moins. Devenue aphorisme, cette citation inexacte et funeste emblématise le statut circulant de la parole littéraire en contexte médiatique, reprise en main par l'auteur journaliste dans l'entretien littéraire, et soumise à d'autres contraintes textuelles dans le cas des interviews d'écrivain.

\section{L'interview d'écrivain : la littérature dépaysée}

Qui a lu les réponses de Zola interviewé sur les chats, la bicyclette, les recalés au "bachot ", ou bien celles de Huysmans répondant à un journaliste l'interrogeant sur «la fermeture d'un bar à filles du quartier latin» (Seillan 2004: 9), ne saurait fondamentalement s'étonner de rencontrer des entretiens avec Orhan Pamuk dans les rubriques non littéraires des titres périodiques.

La particularité de ces articles tient à la posture paradoxale adoptée par le romancier, qui fait de l'interview périodique le lieu où se rejoue la tension entre politique et littérature. Cette dialectique du littéraire et du politique prend place dans un format textuel bien différent de l'entretien littéraire; l'interview d'écrivain organise plus clairement la répartition des paroles respectives de l'écrivain et du journaliste. 


\section{1 Formats professionnalisés}

les codes de l'alternance énonciative, où la parole de l'écrivain se voit toutefois sollicitée sur des questions parfois étrangères au littéraire. Dans Télérama, le 2 avril 2003, Orhan Pamuk est sollicité dans le cadre d'un dossier intitulé "Signes du temps. Guerre en Irak»; dans Le Monde, le 2 avril 2004, lors d'un entretien titré «Il faut débattre d'une définition de l'Europe ", l'écrivain répond à des questions portant sur «l'actualité, en France et en Turquie ». On l'y questionne sur la loi réglementant le port du voile en France, ou sur la candidature turque à l'intégration européenne; dans L'Express, le 3 mai 2007, le journaliste François Busnel lui demande si «Istanbul est soluble dans l'Europe». Dernier exemple, le $n^{\circ} 17$ (hiver 2012) de la revue XXI, périodique trimestriel mêlant bande dessinée, reportage et illustration, confie à Guillaume Perrier, correspondant du Monde à Istanbul, une longue interview avec Orhan Pamuk; intitulé "La force de suggestion des mots et des objets", cet article est complété par de classiques «bonus » journalistiques - cartes, encarts, bibliographie qui dotent cet entretien d'une plus value documentaire proprement informative. Dans tous ces exemples, Orhan Pamuk se trouve être le seul écrivain sollicité pour témoigner d'une réalité lointaine et complexe, celle de la Turquie contemporaine.

Tous ces articles se caractérisent par le respect des normes professionnalisées de l'interview journalistique : les questions posées y sont toujours explicites, rapportées en caractères gras, et d'une brièveté exemplaire. Surtout, on retrouve la combinaison d'effacement énonciatif et d'autorité professionnelle qui caractérise la posture journalistique telle que décrite dans les manuels précédemment cités. La personne du journaliste s'absente, et c'est l'incarnation collective du titre de presse qui prend la parole à sa place: la mention "Télérama " précède chaque question posée dans l'interview d'avril 2003 ; François Busnel, dans L'Express, comme Guillaume Perrier dans XXI, évitent soigneusement toute formule impliquant un pronom personnel.

Enfin, le régime d'actualité de ces interviews ne souffre pas de concession: elles prennent sens dans le moment de leur parution - quel est l'apport aujourd'hui, sinon pour une analyse historique, d'une réflexion analysant la réaction turque à la deuxième guerre en Irak, ou à la loi réglementant le port du voile en France?

Si la parole de l'écrivain trouve dans ce second corpus une place raisonnée, c'est au prix d'un déplacement thématique qui impose à l'actualité du littéraire celle, bien plus large, du politique. Ce sont pourtant les croisements entre ces deux champs qui ont assuré à l'écrivain la place qu'il occupe dans le système de valeurs occidental, depuis la Révolution française (voir Chartier 1990); et toute l'ambiguïté de la posture adoptée par Orhan Pamuk tient à cette intrication à la fois féconde et périlleuse.

\subsection{Figures de la dénégation} février 2005 déclenche, on l'a dit, des réactions en chaîne qui bouleversent son existence, et déterminent une attitude ambivalente vis-à-vis des sollicitations médiatiques.

Avant 2005, l'écrivain paraît jouer sans réticence le rôle de «l'intellectuel engagé » (la formule apparaît dans plusieurs chapeaux en tête d'interviews figurant dans notre 
corpus) ou de porte-parole d'une Turquie occidentalisée, candidate à l'intégration européenne. "Je souhaite que la Turquie intègre l'UE", déclare Pamuk dans un entretien avec Nicolas Monceau, publié dans Le Monde le 2 avril $2004:$ «j'aimerais que les Européens adoptent une position au sujet de leur idée de l'Europe alors que la Turquie frappe à sa porte». L'exergue du roman Neige, traduit en français et en allemand en 2005, et à l'origine indirecte des malheurs de l'écrivain, reprend en la réécrivant la célèbre citation de Stendhal: "La politique dans une œuvre littéraire, c'est un coup de pistolet au milieu d'un concert, quelque chose de grossier et auquel pourtant il n'est pas possible de refuser son attention. Nous allons parler de fort vilaines choses ». À la sortie du roman, circule comme une antienne, d'un entretien à l'autre, cette formule assurant que Neige sera «son premier et son dernier roman politique ».

Après 2005, mis en danger par ses déclarations dans la presse, Pamuk souhaite se replier sur la littérature, « ressaisir la trame du songe » (Le Monde, entretien du 12 mai 2006), revenir à la seule responsabilité dont il se sente investi, «celle de continuer à écrire des livres" (L'Express, 3 mai 2007) : "je considère dorénavant que mon unique responsabilité est de retrouver ma juvénile irresponsabilité et de revenir à la vie que j'avais avant, tournée tout entière vers l'art ", lit-on dans Le Monde (entretien publié le 14 octobre 2006). Pourtant, c'est bien le sort que lui réservent ses adversaires politiques, en raison de ses positionnements politiques, et donc des interviews leur ayant donné une publicité inédite, qui transforme radicalement la notoriété de l'écrivain.

Protégé par les instances internationales, instrumentalisé même dans les négociations autour de l'adhésion turque à l'Union européenne, le cas Pamuk est édifié en symbole de la défense de la liberté d'expression. Un an après la publication de l'entretien dans le journal suisse, le prix Nobel de littérature vient compléter la liste déjà impressionnante des prix reçus par l'écrivain (prix du meilleur livre étranger à New York, prix Médicis en France, prix de l'Union des libraires allemands); il s'agit, précise l'Académie suédoise, de distinguer un écrivain «qui a trouvé de nouvelles images spirituelles pour le combat et l'entrelacement des cultures ${ }^{7}$.

47 Le dépaysement du littéraire dans les pages des journaux trouve donc pour symétrique cette récompense ambiguë, qui célèbre à la fois une œuvre littéraire (" ses images spirituelles ») et les combats culturels, voire politiques, menés par son auteur. Dès lors, les interviews sont le lieu pour Pamuk d'une constante remise en jeu de la valeur autonome ou hétéronome de la littérature - remise en jeu qui passe par une pratique constante de la dénégation.

48 «L'écrivain qui reçoit le prix Nobel n'est-il pas chargé d'une plus grande responsabilité ?» lui demande François Busnel dans L'Express, le 3 mai 2007 : «C'est un malentendu », répond Orhan Pamuk, qui précise avoir « reçu le prix Nobel » « pas pour sauver le monde ou le changer ", mais pour "continuer à écrire des livres». "Vous aviez parlé de 'ce pays où l'on a tué un million d'Arméniens et trente mille kurdes' », lui rappelle Guillaume Perrier en 2012 dans l'article de la revue XXI : "Je ne veux pas revenir sur cet épisode» répond le romancier. «Mais vous dites que la Turquie a changé », insiste le journaliste : «La Turquie a changé, mais mon histoire personnelle ne correspond pas à ce constat », élude encore l'écrivain.

49 Il y a donc deux Pamuk, pourrait-on conclure à la lecture de ces articles : d'une part l'écrivain de stature internationale, édifié en symbole, célébré tout à la fois pour ses 
livres et pour son engagement politique, et d'autre part le romancier absorbé dans la fabrication de son œuvre, qui ne voit le monde que par la fenêtre de son bureau stambouliote. Au lendemain de l'affaire Dreyfus, quand la figure de l'intellectuel est née de l'engagement des écrivains dans un combat à la fois médiatique et politique, on s'interrogeait en des termes proches sur l'identité double d'un Zola candidat posthume à la panthéonisation : « ce n'est pas au romancier Zola, c'est au citoyen Zola qu'il faut accorder les honneurs du Panthéon ", déclarait par exemple Jean Jaurès dans un article paru à la Neue Freie Press en janvier $1908^{8}$.

\section{Conclusion}

Interview d'écrivain et entretien littéraire : ces expressions désignent à notre sens deux modèles permettant de penser les possibilités offertes à la mise en texte périodique d'un dialogue entre écrivain et journaliste. Autour de la figure d'Orhan Pamuk, les exemples d'interviews sont assez nombreux pour manifester la souplesse des formes explorées par les supports médiatiques; nous n'en avons retenu qu'une partie, aux fins de la démonstration. Entre les deux modalités éditoriales qui nous ont fourni notre cadre d'analyse, s'intercalent des exemples qui ont tous leurs singularités. S'y donne à lire toutefois une même tension entre figure du journaliste et figure de l'écrivain, qui a fortement à voir, dans le cas examiné, avec l'articulation entre actualité littéraire et actualité politique. Alors que l'entretien d'écrivain accorde une place dominante à la figure du romancier, et à son engagement sur la scène publique, l'entretien littéraire, lui, entremêle les voix des interlocuteurs, plongeant au cœur de la fabrique de l'œuvre, tout en faisant la part belle à la plume du journaliste. L'entretien littéraire, espace crucial de la médiatisation des écrivains, s'avère donc l'un des lieux où se met en œuvre une délibération quotidienne sur les pouvoirs de la littérature.

\section{BIBLIOGRAPHIE}

Amossy, Ruth \& Dominique Maingueneau (éds). 2004. L'Analyse du discours dans les études littéraires (Toulouse : Presses universitaires du Mirail)

Amossy, Ruth, 2009. « La double nature de l'image d'auteur », Argumentation et analyse du discours 3 [En ligne : http://aad.revues.org/662]

Chartier, Roger, 1990. Les Origines culturelles de la Révolution française (Paris : Seuil)

Duclert, Vincent. 2008. Alfred Dreyfus. L'honneur d'un patriote (Paris : Fayard)

Lavaud, Martine \& Marie-Ève Thérenty. 2006. « Avant-propos », Lieux littéraires 9-10 (L'interview d'écrivain), 7-25

Lopez Munoz, Juan-Manuel \& Francisca Romeral Rosel. 2006. « Discours permanents, discours en co-énonciation et en écho-énonciation dans les entretiens : la pratique de l'auto-citation chez Annie Ernaux », Travaux de linguistique 52, 85-100 
Maingueneau, Dominique. 2009. " Auteur et image d'auteur en analyse du discours ", Argumentation et analyse du discours 3 [En ligne : http://aad.revues.org/660?lang=en]

Maingueneau, Dominique. 2012. Les Phrases sans texte (Paris : Colin)

Montant, Henri. 1995. L'Interview écrite et le portrait. (Paris : Guides du CFPJ)

Rémond, Edith. 2007. L'Interview (Paris : Victoire éditions)

Ringoot Roselyne \& Yvon Rochard. 2007. « Proximité éditoriale : normes et usages des genres journalistiques », Mots 77, 73-90

Seillan, Jean-Marie. 2002. Interviews de Joris-Karl Huysmans (Paris : Champion)

Seillan, Jean-Marie. 2004. « Identité générique et contraintes éditoriales : l'exemple de l'interview littéraire à la fin du $19^{e}$ siècle », Loxias 4 [En ligne : http://revel.unice.fr/loxias/?id=31]

Souchier, Emmanuël. 1998. "L'image du texte : pour une théorie de l'énonciation éditoriale », Cahiers de médiologie 2, 136-46

Speirs, Dorothy. 1990. «Un genre résolument moderne : l'interview », Romance quarterly 3-4, 301-309

Yanoshevsky, Galia. 2006. Les Discours du nouveau roman : essais, entretiens, débats. (Villeneuve d'Ascq : Presses universitaires du Septentrion)

Zola, Émile. 1893. « M. Zola interviewé sur l'interview », Le Figaro, entretien avec Émile Leyret, 12 janvier 1893

\section{NOTES}

1. Voici la liste complète de ces articles, dans l'ordre chronologique de leur parution : Libération, «La démarche turque», Jean-Baptiste Harang, 14 janvier 1999; Télérama, "Orhan Pamuk, romancier turc. Le peuple tient les Américains pour responsables de sa misère », propos recueillis par Christian Sorg, 2 avril 2003; Le Monde «Il faut débattre d'une définition de l'Europe", "Orhan Pamuk, écrivain turc et citoyen engagé », propos recueillis par Nicolas Monceau, 2 avril 2004 ; L'Express, « 3 questions à Orhan Pamuk », propos recueillis par Nükte V. Ortaq, 13 décembre 2004 ; Le Monde, "Orhan Pamuk, une conscience turque», propos recueillis par Catherine Bedarida, 4 avril 2005 ; L'Humanité, «La durée d'un flocon », entretien à propos de Neige, propos recueillis par Alain Nicolas, 6 octobre 2005 ; Le Monde, "Orhan Pamuk : être un artiste libre", propos recueillis par Lila Azam Zanganeh, 12 mai 2006 ; Le Monde, "Mon unique responsabilité : revenir avec la vie que j'avais avant ", extraits inédits de l'entretien du 12 mai 2006 avec Lila Azam Zanganeh publiés le 14 octobre 2006 dans une page consacrée à « Orhan Pamuk, prix Nobel de littérature »; Le Figaro, «La semaine suédoise d'Orhan Pamuk », Clémence Boulouque, 9-10 décembre 2006 ; L'Express, « Orhan Pamuk : je n'écris pas pour changer le monde », entretien avec François Busnel, 3 mai 2007 ; Le Point, «Pamuk, un Nobel en danger», propos recueillis par François-Guillaume Lorrain, 10 mai 2007 ; Télérama, « Un désir agressif d'écrire », Nathalie Crom, 25 juillet 2007 ; Le Nouvel Observateur, «L'Europe, une passion turque, par Orhan Pamuk », propos recueillis par François Armanet et Gilles Anquetil, 22 octobre 2009 ; Le Monde, « Orhan Pamuk : la vie se moque de mes romans ", rencontre avec Xavier Houssin, 24 avril 2011 ; Libération, "Orhan Pamuk dans la famille Iletsim », par Marc Semo, 8 août 2011 ; XXI, « La force de suggestion des mots et des objets. Orhan Pamuk», propos recueillis par Guillaume Perrier, nº17, hiver 2012 ; Le Monde, «Le musée aux mille pages », par Guillaume Perrier, 28 avril 2012; Le Figaro, "Orhan 
Pamuk, un musée entre rêve et réalité, entretien avec Laure Marchand, 3 mai 2012 ; Le Magazine littéraire, « La vigie myope d'Istanbul », par Jean-Baptiste Harang, août 2013.

2. Citation d'un article du Temps, repris par Jean-Marie Seillan dans sa «Préface » aux Interviews de Huysmans (2002: 16)

3. «Man hat hier 30000 Kurden umgebracht. Und eine Million Armenier. Und fast niemand traut sich, das zu erwähnen ", Das Magazin, 5 février 2005.

4. Respectivement, deux articles dans Libération, «La démarche turque », Jean-Baptiste Harang, 14 janvier 1999 et « Orhan Pamuk dans la famille Ilestsim », 8 août 2011 ; un article dans Télérama, «Un désir agressif d'écrire », Nathalie Crom, 25 juillet 2007 ; trois articles dans Le Monde, "Orhan Pamuk, une conscience turque ", Catherine Bedarida, 4 novembre 2005, "Orhan Pamuk : la vie se moque de mes romans ", Xavier Houssin, 24 avril 2011, et "Un musée aux mille visages ", Guillaume Perrier, 28 avril 2012; un article dans Le Figaro, «La semaine suédoise d'Orhan Pamuk », Clémence Boulouque, 9-10 décembre 2006

5. Voir le site à l'adresse suivante : www.leboutdelalangue.com

6. Nous traduisons.

7. Communiqué de presse du prix Nobel 2006, consultable sur le site www.nobelprize.org (http://www.nobelprize.org/nobel_prizes/literature/laureates/2006/press_fr.html?print=1).

8. Cité par Vincent Duclert, dans sa biographie d'Alfred Dreyfus (Duclert 2006 : 980)

\section{RÉSUMÉS}

Cet article analyse le rôle joué par les entretiens littéraires dans l'édification et la mise en circulation des images d'auteur. Le cas choisi est celui de l'écrivain turc Orhan Pamuk, prix Nobel de littérature en 2006, traduit en France chez Gallimard, et interlocuteur régulier des journalistes français. À partir d'une typologie distinguant entretiens d'écrivains et interviews littéraires, l'analyse met en relation les modes de textualisation et d'éditorialisation de la parole écrite avec la réactivation d'une rivalité latente entre l'écrivain et le journaliste. Dix-neuf articles sont ici analysés, qui montrent à quel point le jeu de forces entre interviewer et interviewé exerce une incidence sur la conception et même l'évolution de la création littéraire, tendue chez Pamuk entre engagement et idéal d'autonomie. À la suite d'un entretien publié en 2005 dans un journal suisse, où il évoque le sort connu par les Arméniens et les Kurdes en Turquie, Pamuk se voit menacé de mort dans son pays; cet événement médiatique lui donne une notoriété paradoxale, et n'est pas sans lien avec l'attribution à son œuvre, en 2006, du prix Nobel de littérature. Différents critères d'analyse sont proposés pour interpréter le fonctionnement médiatique de l'entretien avec un écrivain : ils concernent notamment les modalités de citation, l'alternance énonciative, le rubriquage, la mise en récit et les jeux typographiques.

The purpose of this paper is to analyze the roles played by literary interviews in the construction and the circulation of the author's images in the Press. By alluding to interviews with Turkish writer and Nobel laureate (2006) Orhan Pamuk, the reader is given to see the struggle of power between the writer and the journalist, which plays a considerable role in the conception and evolution of literary creation. This conception is situated for Pamuk between engagement and an ideal of autonomy. Based on the assumption that there exists a relationship between the attribution of a lucrative literary prize and Pamuk's political notoriety (following his 2005 interview where he mentions the Turkish treatment of Armenians and Kurds in Turkey), this 
paper suggests various criteria for the interpretation of the interview's communicational mechanisms such as the modalities of citation, verbal interaction, choice of rubrics, narration and typography.

INDEX

Keywords : author, interview, Pamuk (Orhan), utterance, writer

Mots-clés : auteur, énonciation, entretien, figure d'écrivain, interview, Pamuk (Orhan)

\section{AUTEUR}

ADELINE WRONA

Université Paris-Sorbonne, GRIPIC - EA1498, CELSA 